
jóvenes universitarios. Cuadernos de Psicología del Deporte, 20(2), 265-275

\title{
Estrés percibido y felicidad auténtica a través del nivel de actividad física en jóvenes universitarios
}

\section{Perceived stress and authentic happiness through the level of physical activity in young university students}

\section{Estresse percebido e felicidade autêntica através do nível de atividade física em jovens estudantes universitários}

\author{
López-Walle, Jeanette1, Tristán, José1, Tomás, Inés2, Gallegos-Guajardo, Julia3, Gongora, Elias4, y \\ Hernández-Pozo, M. del Rocío5 \\ 1 Universidad Autónoma de Nuevo León, México;2Universitat de València, España; 3 Universidad de \\ Monterrey, México; 4 Universidad Autónoma de Yucatán, México; 5 Universidad Nacional Autónoma de \\ México, México
}

\begin{abstract}
RESUMEN
Se ha demostrado en la literatura que el estrés y la felicidad se han relacionado de diversas maneras, y estas a su vez, también se relacionan con la práctica de actividad física (AF). El objetivo de este trabajo fue poner a prueba el papel de la AF en la reducción del estrés y el aumento de la felicidad, así como su rol mediador en la relación entre ambas variables. Concretamente, se pusieron a prueba las siguientes hipótesis: 1) la percepción del estrés (con o sin control de la situación estresante) y la felicidad auténtica difieren en función del nivel de AF; 2) a mayor práctica de AF, mayor percepción de control en la situación estresante y menor percepción de sentirse sobrepasado por la situación; 3) a mayor práctica de AF mayor felicidad auténtica; y 4) la relación entre la percepción del estrés (con o sin control de la situación estresante) y la felicidad auténtica está mediada por la AF. Participaron 938 estudiantes de licenciatura, con un rango de edad de 17 a 51 años $(M=20.25, D T=3.34), 521$ mujeres y 417 hombres. Respecto a la actividad física (AF) que realizan: $15.1 \%$ no realiza ninguna actividad; $19.7 \%$ realiza actividad irregular; $28 \%$ actividad física moderada (2hrs/sem); y 37.1\% actividad intensa (20min/día, 5 veces/sem). Los participantes contestaron la PSS (Cohen, Kamarck, y Mermelstein, 1983) y el AHI (Shepherd, Oliver, y Schofield, 2015). Mediante análisis descriptivos, ANOVAs y modelos de regresión mediada se dio respuesta a las hipótesis. Los resultados muestran que la percepción del estrés (con o sin control de la situación estresante) y la felicidad auténtica difieren significativamente en función del nivel de AF. Concretamente, a mayor nivel de práctica de AF mayor percepción de control ante una situación estresante y mayor felicidad auténtica; mientras que a menor práctica de AF, mayor percepción de verse sobrepasado ante una situación estresante. Por último, el nivel de actividad física media la relación entre el estrés percibido (con control de la situación estresante y sin control de la situación estresante) y la felicidad auténtica. Con base en los resultados concluimos que la $\mathrm{AF}$ ayuda a percibir un menor estrés ante situaciones demandantes, por lo que promueve un bienestar psicológico.
\end{abstract}

Palabras clave: bienestar psicológico, estrés percibido, felicidad, actividad física. 


\title{
López-Walle, J., Tristán, J., Tomás, I., Gallegos-Guajardo, J., Gongora, E., y Hernández-Pozo, M. R.
}

\begin{abstract}
Previous literature has shown that stress and happiness are related in various ways, and both variables have been also related to physical activity (PA) practice. The objective of the present study was to test the role of PA in the decrement of stress and increment of happiness, and additionally to test its mediator role in the relationship between the two aforementioned variables. Concretely, the following hypotheses were tested: 1) the perceived stress (with or without control of the stressful situation) and the authentic happiness differ across different levels of PA; 2) subjects with higher practice of PA will show higher perceived control and lower loss of control in stressful situations; 3) subjects with higher practice of PA will show higher authentic happiness; and 4) the relationship between perceived stress (with or without control of the stressful situation) and authentic happiness is mediated by PA. Participants were 938 undergraduate students from different careers, with an age range from 17 to 51 years $(M=20.25, S D=3.34), 521$ women and 417 men. Regarding physical activity (PA) practice: $15.1 \%$ does not to do any activity; $19.7 \%$ does an irregular PA; $28 \%$ does moderate PA (2hrs / week); and 37.1\% does intense PA (20min/day, 5 times/week). Participants answered the PSS (Cohen, Kamarck, y Mermelstein, 1983) and the AHI (Shepherd, Oliver, y Schofield, 2015). We used descriptive analysis, ANOVAs and mediated regression models to test the hypotheses. Results showed that perceived stress (with or without control of the stressful situation) and authentic happiness differ significantly across PA levels. Concretely, higher level of PA practices was related to higher perception of control in a stressful situation and to higher authentic happiness; otherwise, the lower the PA practice, the greater the perception of being overwhelmed by a stressful situation. Finally, the level of physical activity mediated the relationship between perceived stress (with and without control of the stressful situation) and authentic happiness. Based on these results, we concluded that PA helps to perceive less stress in demanding situations, thus promoting psychological well-being. Keywords: psychological well-being, perceived stress, happiness, physical activity.
\end{abstract}

\section{RESUMO}

A literatura anterior mostrou que o estresse e a felicidade estão relacionados de várias maneiras, e ambas as variáveis também estão relacionadas à prática de atividade física (AF). O objetivo do presente estudo foi testar o papel da $\mathrm{AF}$ no decréscimo do estresse e no incremento da felicidade, além de testar seu papel mediador na relação entre as duas variáveis mencionadas. Concretamente, foram testadas as seguintes hipóteses: 1) o estresse percebido (com ou sem controle da situação estressante) e a felicidade autêntica diferem nos diferentes níveis de AF; 2) sujeitos com maior prática de AF mostrarão maior controle percebido e menor perda de controle em situações estressantes; 3) sujeitos com maior prática de AF mostrarão maior felicidade autêntica; e 4) a relação entre o estresse percebido (com ou sem controle da situação estressante) e a felicidade autêntica é mediada pela AF. Participaram 938 estudantes de graduação de diferentes carreiras, com faixa etária de 17 a 51 anos $(M=20,25, T D=3,34), 521$ mulheres e 417 homens. Em relação à prática de atividade física (AF): $15,1 \%$ não realiza nenhuma atividade; $19,7 \%$ faz AF irregular; $28 \%$ moderam AF (2 horas / semana); e 37,1\% realizam AF intensa (20min / dia, 5 vezes / semana). Os participantes responderam ao PSS (Cohen, Kamarck, e Mermelstein, 1983) e ao AHI (Shepherd, Oliver, e Schofield, 2015). Utilizamos análise descritiva, ANOVAs e modelos de regressão mediada para testar as hipóteses. Os resultados mostraram que o estresse percebido (com ou sem controle da situação estressante) e a felicidade autêntica diferem significativamente entre os níveis de AF. Concretamente, um nível mais alto de práticas de AF estava relacionado à maior percepção de controle em uma situação estressante e à maior felicidade autêntica; caso contrário, quanto menor a prática de AF, maior a percepção de ser sobrecarregado por uma situação estressante. Por fim, o nível de atividade física media a relação entre o estresse percebido (com e sem controle da situação estressante) e a felicidade autêntica. Com base nesses resultados, concluímos que a AF ajuda a perceber menos estresse em situações exigentes, promovendo o bem-estar psicológico.

Palavras chave: bem-estar psicológico, estresse percebido, felicidade, atividade física.

\section{INTRODUCCIÓN}

En la psicología positiva se destacan las fortalezas de los individuos, las características y las acciones que les permiten trascender tanto a ellos como a su contexto, basada en la creencia de que las personas quieren llevar una vida plena y con sentido, que les provoque 


\section{Estrés, felicidad y actividad física en jóvenes universitarios}

mayores experiencias positivas y relaciones más satisfactorias (Seligman, 2013). Diener y Chan (2011) mencionan diversos factores que frecuentemente se incluyen para enumerar las características de una buena vida, entre ellas la felicidad y la salud, lo cual está muy relacionado.

Dentro de la psicología positiva se destaca el concepto de bienestar subjetivo, el cual es definido como las evaluaciones cognitivas y afectivas que una persona hace en torno a su vida. Estas evaluaciones incluyen tanto reacciones emocionales a acontecimientos, así como juicios sobre satisfacción y logro. El bienestar subjetivo es pues, un concepto amplio que incluye la experiencia de emociones agradables o positivas, ausencia o bajo nivel de emociones negativas y alto nivel de satisfacción con la vida (Diener, 2000). Sin embargo, hay autores que consideran que estos dos conceptos son diferentes (bienestar subjetivo y felicidad), siendo el de felicidad más amplio y complejo (Alarcón, 2006). Por ejemplo, Quick y Quick (2004) la definen como un estado holístico de bien-estar y bien-hacer. Así, la felicidad surgiría no solo de la experimentación de bienestar sino también de la idea de estar haciendo bien las cosas (Seligman, 2011). Por lo que la felicidad auténtica sería el grado en el que la persona posee un estado de ánimo positivo, considera que obtiene logros importantes y gratificantes y le encuentra sentido a su vida (Schiffrin y Nelson, 2010).

La felicidad se considera a menudo como uno de los objetivos más importantes de la vida y en las últimas décadas se ha visto una explosión de investigaciones sobre la felicidad. Numerosas teorías y estudios científicos han intentado identificar las causas de la felicidad a partir de diversos factores (Cheng y Furmham, 2001; Dambrun et al., 2012; Hills y Arglye, 2001; Scorsolini-Comin, Fontaine, Koller, y dos Santos, 2013; Veenhoven y Ehrhard, 1995).

Comúnmente podría considerarse que el estrés impide la felicidad, por lo que su asociación ha sido analizada de diversas maneras en la literatura. En el estudio realizado por Schiffrin y Nelson (2010) demostraron en estudiantes universitarios la relación inversa entre el estrés percibido y la felicidad. Asimismo, Terrill et al. (2014) encontraron una relación inversa entre el distress (estrés negativo) y la felicidad. Las intervenciones dirigidas tanto al estrés como a la felicidad han demostrado que las actividades como el ejercicio, la meditación y la expresión escrita, disminuyen el estrés al mismo tiempo que aumentan la felicidad (Compton, 2005; King 2001; Lyubomirsky, Sousa, y Dickerhoof, 2006). Pero los efectos de estas actividades se han asociado principalmente en términos de los aspectos fisiológicos del estrés y no desde la perspectiva cognitiva de la percepción del estrés.

La felicidad también se ha relacionado con la práctica de actividad física (AF). En un estudio transcultural de 15 países europeos se analizó la asociación entre la actividad física y la felicidad. La conclusión a la que se llegó fue que a mayor volumen de actividad física mayores niveles de felicidad (Richards et al., 2015). Otro estudio de Jimenez-Moral, Zagalaz, Molero, Pulido-Martos y Ruiz (2013) indicaron que la capacidad aeróbica, medida con el test de los 20 metros, está asociada de forma positiva con la felicidad. En la misma línea, Piqueras, Kuhne, VeraVillarroel, van Straten, y Cuijpers (2011) demostraron que las personas que informaban realizar $\mathrm{AF}$ diariamente se clasificaban como más felices; mientras que los que se sentían estresados en situaciones normales y de prueba, mostraron menor probabilidad de sentirse felices. Por último, en el estudio de Opheim, Eriksen, Moksnes, y Espnes (2011) se asoció la actividad física, el estrés y la felicidad en adolescentes, resultando que, quienes informaron haber participado en actividad física de 2 a 3 veces por semana o más, obtuvieron puntuaciones significativamente más bajas en el estrés y más altas en la felicidad, comparados con aquellos que participan en actividad física un día por semana o menos. No hubo una diferencia significativa en el estrés y la felicidad entre los que hacían AF dos o tres veces a la semana y los que la hacían diariamente.

Con base en lo anterior, se ha confirmado la relación entre el estrés percibido y la felicidad, el nivel de actividad física y la felicidad, pero aún no se ha indagado acerca de la asociación entre la felicidad y la percepción del control ante una situación estresante, o al contrario, verse sobrepasado por la situación estresante. Tampoco se ha encontrado en la literatura ningún estudio que explore el papel mediador que el nivel de actividad física pudiera tener en la relación entre el estrés percibido y la felicidad. Por lo cual, en este estudio el objetivo fue poner a prueba el papel de la $\mathrm{AF}$ en la reducción del estrés y el aumento de la 


\section{López-Walle, J., Tristán, J., Tomás, I., Gallegos-Guajardo, J., Gongora, E., y Hernández-Pozo, M. R.}

felicidad, así como su rol mediador en la relación entre ambas variables. Concretamente, se pusieron a prueba las siguientes hipótesis: 1) la percepción del estrés (con o sin control de la situación estresante) y la felicidad auténtica difieren en función del nivel de AF; 2) a mayor práctica de $\mathrm{AF}$, mayor percepción de control en la situación estresante y menor percepción de sentirse sobrepasado por la situación; 3) a mayor práctica de $\mathrm{AF}$, mayor felicidad auténtica; y 4) la relación entre la percepción del estrés (con o sin control de la situación estresante) y la felicidad auténtica está mediada por la AF.

\section{MATERIAL Y MÉTODOS}

\section{Participantes}

En este estudio participaron un total de 938 estudiantes de licenciatura de las carreras de enfermería $(n=103)$, psicología $(n=575)$ y ciencias del ejercicio $(n=260)$, con un rango de edad de 17 a 51 años $(M=20.25, D T$ $=3.34$ ), 521 mujeres y 417 hombres, el $95.3 \%$ su estado civil es soltero.

Respecto a la actividad física que realizan: $15.1 \%$ no realiza ninguna actividad; $19.7 \%$ realiza actividad irregular; $28 \%$ actividad física moderada ( $2 \mathrm{hrs} / \mathrm{semana}) ;$ y $37.1 \%$ actividad intensa (20min/día, 5 veces/semana).

\section{Instrumentos}

Para evaluar la felicidad auténtica, se utilizó el Inventario de Felicidad Auténtica (Authentic Happiness Inventory, AHI, Shepherd, Oliver, y Schofield, 2015) adaptado al contexto mexicano (Hernández-Pozo et al., en revisión-b). El inventario evalúa los cambios en las emociones positivas basadas en la felicidad, el compromiso y el significado de la vida. Consta de 24 ítems en donde los encuestados deben seleccionar de 1 a 5 opciones acerca de los sentimientos que han tenido en la última semana, incluido el día que contestan la encuesta. Ningún ítem tiene puntaje inverso. Un ejemplo de ítem es "Durante la última semana incluyendo hoy..." 1 . He estado muy triste; 2. No he estado ni triste, ni tampoco alegre; 3. Ha habido más dicha que tristeza; 4. Ha habido mucha más dicha que penas; 5 . He estado lleno/a de dicha.

La escala ha mostrado adecuada fiabilidad en Alemania (Proyer, Gander, Wellenzohn, y Ruch,
2017), Colombia (Sanín-Posada y Salanova, 2015), Irán (Zabihi, Ketabi, Tavakoli, y Ghadiri, 2014) y Nueva Zelanda (Shepherd et al., 2015).

Para evaluar el estrés percibido se utilizó la Escala de Percepción del Estrés (Perceived Stress Scale, PSS, Cohen, Kamarck, y Mermelstein, 1983) adaptado al contexto mexicano (Hernández-Pozo et al., en revisión-a). La escala evalúa la frecuencia en la aparición de sentimientos y pensamientos ante diversas situaciones estresantes presentadas durante el último mes. La escala consta de 14 ítems, distribuidos en dos factores: Control de la situación (7 ítems), por ejemplo, "¿Con qué frecuencia sentiste haber manejado con éxito los pequeños problemas irritantes de la vida?"; Sobrepasado por la situación (7 ítems), por ejemplo, “¿Con qué frecuencia estuviste molesto/a por algo que ocurrió inesperadamente?". Las respuestas son de tipo Likert en una escala que oscila del 0 que representa nunca a 4 que representa a menudo.

La escala ha mostrado adecuadas propiedades psicométricas con estudiantes mexicanos (González y Landero, 2007), colombianos (Campo-Arias, BustosLeiton, y Romero-Chaparro, 2009), turcos (Örücü y Demir, 2009), griegos (Andreou et al., 2011) y en otros contextos (Lee, 2012; Remor, 2006).

La validez de constructo se obtuvo con la medida de adecuación muestral de Kaiser-Meyer-Olkin (KMO) de .970 (prueba de esfericidad de Bartlett $\mathrm{c}_{2}=$ 18808.72, $g l=703, p<.001)$, confirmando tres componentes que explican el $51.24 \%$ de la varianza acumulada. El Componente 1 (Felicidad auténtica) representado por 24 ítems con puntuaciones factoriales que oscilan de .31 a .82 ; Componente 2 (Control de la situación estresante) representado por 7 ítems con puntuaciones factoriales que oscilan de .51 a .76; y Componente 3 (Sobrepasado por la situación estresante) representado por 7 ítems con puntuaciones factoriales que oscilan de .57 a .79 .

La actividad física se evaluó mediante la pregunta ¿ $\mathrm{Su}$ actividad física por lo regular es?, teniendo cuatro opciones de respuesta: 1, ninguna; 2, irregular; 3 , moderada (2hrs/semana); e intensa (20min/día, 5 veces/semana). 


\section{Estrés, felicidad y actividad física en jóvenes universitarios}

Los gradientes de respuesta son similares a los utilizados en otro estudio (Dias, Corte-Real, Corredeira, Barreiros, Bastos y Fonseca, 2008).

\section{Procedimiento}

Las aplicaciones de los cuestionarios se realizaron en versión electrónica mediante la aplicación de Google Forms. La participación fue voluntaria y anónima, aceptando Consentimiento Informado, el cual mencionaba el objetivo del estudio, la duración aproximada de aplicación, que no constituye un riesgo para la salud física y mental, y la confidencialidad de los datos. Se gestionaron con las autoridades los permisos y calendarizaciones para la programación de las aplicaciones en las aulas de cómputo de las diversas instituciones educativas.

\section{Análisis estadístico}

Las aplicaciones de los cuestionarios se realizaron en versión electrónica mediante la aplicación de Google Forms. La participación fue voluntaria y anónima, aceptando Consentimiento Informado, el cual mencionaba el objetivo del estudio, la duración aproximada de aplicación, que no constituye un riesgo para la salud física y mental, y la confidencialidad de los datos. Se gestionaron con las autoridades los permisos y calendarizaciones para la programación de las aplicaciones en las aulas de cómputo de las diversas instituciones educativas.

\section{Procesamiento de datos}

Se llevaron a cabo los siguientes análisis mediante el software SPSS v. 24. 1) Descriptivos de tendencia central (media), dispersión (desviación típica y rango), y prueba de normalidad Kolmogorov-Smirnov (K-S) de las variables de estrés percibido (con y sin control de la situación estresante) y felicidad auténtica; 2) Consistencia interna de los instrumentos por variable, mediante el alfa de Cronbach, siguiendo los siguientes criterios, propuestos por George y Mallery (2001):
Coeficiente Alfa $>.90$ excelente,>.80 bueno,>.70 aceptable, $>.60$ cuestionable, $>.50$ es pobre y $<.50$ inaceptable; 3) Análisis de Varianza para identificar diferencias en estrés percibido y felicidad auténtica en función del nivel de práctica de AF; 4) para conocer el tamaño del efecto, se utilizó la eta cuadrado $\left(\eta_{2}\right)$, considerando la clasificación de Cohen (1988): $\eta_{2}$ $<.01$ despreciable, $.01<\eta_{2}<.06$ bajo, $.06<\eta_{2}<.14$ medio, $\eta_{2}>.14$ grande; 5) Los modelos de mediación fueron estimados mediante análisis de regresión con la macro PROCESS (Hayes, 2013) para SPSS. Para poner a prueba los efectos indirectos, utilizamos intervalos de confianza bootstrap corregidos de sesgo, con 5000 replicaciones y nivel de confianza del $95 \%$. Este método implica calcular el producto de los coeficientes de regresión que estiman el efecto indirecto, y obtener un intervalo de confianza para dicho efecto. Si el intervalo de confianza no incluye el valor cero, se confirma el efecto de mediación (Hayes, 2013).

\section{RESULTADOS}

\section{Análisis descriptivos y fiabilidad}

En primera instancia se muestra la media y desviación típica de las variables de percepción del estrés, siendo mayor el promedio obtenido en el factor de control de la situación estresante $(M=2.70)$, comparado con el factor sobrepasado por la situación $(M=1.96)$.

La consistencia interna de las puntuaciones obtenidas con los cuestionarios utilizados resultó ser satisfactoria, con valores de alfa de Cronbach que oscilan entre .85 y .95 . Las interrelaciones entre las variables denotaron una asociación positiva y significativa entre la percepción del estrés con control de la situación estresante y la felicidad auténtica ( $r$ $=.55, p<.01)$, mientras que la relación entre percepción del estrés sintiéndose sobrepasado por la situación estresante y felicidad auténtica fue negativa $(r=-.39, p<.01)($ ver Tabla 1$)$.

Tabla 1. Análisis descriptivo, fiabilidad y correlación entre variables

\begin{tabular}{llllllllll}
\hline Variables & Rango & $M$ & $D T$ & Asi & Cur & K-S & $\alpha$ & 1 & 2 \\
\hline $\begin{array}{l}\text { 1. Control de la situación estresante } \\
\text { 2. Sobrepasado por la situación }\end{array}$ & $0-4$ & 2.70 & .66 & -.39 & -.09 & $.08^{* *}$ & .85 & - & \\
estresante & $0-4$ & 1.96 & .73 & .18 & -.07 & $.06 * *$ & .82 & $-.33^{* *}$ & - \\
3. Felicidad Auténtica & $1-5$ & 3.19 & .69 & .23 & -.33 & $.04 *$ & .95 & $.55^{* *}$ & $-.39 * *$ \\
\hline
\end{tabular}

Nota. Asi $=$ Asimetría; Cur $=$ Kurtosis. ${ }^{*} p<.01, * * p<.001$ 


\section{López-Walle, J., Tristán, J., Tomás, I., Gallegos-Guajardo, J., Gongora, E., y Hernández-Pozo, M. R.}

Diferencias en las variables en función del nivel de práctica deportiva

En la Tabla 2 se muestran los resultados de los ANOVAs en donde se observan diferencias significativas en las variables de percepción del estrés (con control de la situación y sobrepasado por la situación) y la felicidad auténtica, en función del nivel de AF. El tamaño del efecto de las diferencias encontradas resultó ser bajo en los tres contrastes. Los resultados de las pruebas de comparaciones a posteriori $(p<.01)$ indicaron que la media en la variable de estrés cuando se percibe control en la situación estresante fue mayor cuando el nivel de AF es intensa $(M=2.80)$, y menor cuando no se realiza $\mathrm{AF}(M=2.59)$, o bien hay una práctica irregular $(M=$
2.63). Respecto a la percepción del estrés en situaciones que le sobrepasan, la media fuer mayor cuando no realiza AF $(M=2.09)$, o lo hace de forma irregular $(M=2.08)$, comparado cuando hace una $\mathrm{AF}$ intensa $(M=1.88)$, obteniendo diferencias estadísticamente significativas entre estos grupos ( $p$ $<$.01). Por último, al igual que ante la percepción del control ante una situación estresante, las pruebas de comparaciones a posteriori $(p<.01)$ indicaron que la media en felicidad auténtica es mayor cuando el nivel de práctica de $\mathrm{AF}$ es intensa $(M=3.29)$, y menor cuando no se realiza AF $(M=3.03)$. En las Figuras 1 a 3 se representa gráficamente la asociación entre las tres variables según el nivel de práctica de actividad física.

Tabla 2. Análisis descriptivo e inferencial por variable según la Actividad Física

\begin{tabular}{|c|c|c|c|c|c|c|c|c|c|c|c|c|c|c|c|}
\hline & \multicolumn{15}{|c|}{ Actividad Física } \\
\hline & \multicolumn{3}{|c|}{$\begin{array}{l}\text { Ninguna } \\
(n=142)\end{array}$} & \multicolumn{3}{|c|}{$\begin{array}{l}\text { Irregular } \\
(n=185)\end{array}$} & \multicolumn{2}{|c|}{$\begin{array}{c}\text { Moderada } \\
(2 \mathrm{hrs} / \mathrm{sem}) \\
(n=263)\end{array}$} & \multicolumn{3}{|c|}{$\begin{array}{c}\text { Intensa } \\
(20 \mathrm{~min} / \mathrm{día}, \\
5 \text { veces/sem }) \\
(n=348)\end{array}$} & \multirow[b]{2}{*}{$K-S$} & \multirow[t]{2}{*}{$F$} & \multirow[t]{2}{*}{$p$} & \multirow[t]{2}{*}{$\eta^{2}$} \\
\hline & $M$ & $D T$ & $K-S$ & $M$ & $D T$ & $K-S$ & $M$ & $D T$ & $K-S$ & $M$ & $D T$ & & & & \\
\hline $\begin{array}{l}\text { Control de la } \\
\text { situación } \\
\text { estresante }\end{array}$ & 2.59 & .63 & .06 & 2.63 & .65 & .04 & 2.66 & .71 & $.06^{*}$ & 2.80 & .66 & $.06^{*}$ & 4.65 & .003 & 0.020 \\
\hline $\begin{array}{l}\text { Sobrepasado } \\
\text { por la } \\
\text { situación } \\
\text { estresante }\end{array}$ & 2.09 & .710 & $.08^{*}$ & 2.08 & .74 & $.09 *$ & 1.91 & .73 & $.08 *$ & 1.88 & .72 & $.09 *$ & 5.06 & .002 & 0.015 \\
\hline $\begin{array}{l}\text { Felicidad } \\
\text { Auténtica }\end{array}$ & 3.03 & .74 & $.08 *$ & 3.09 & .63 & $.08 *$ & 3.20 & .69 & $.07 *$ & 3.29 & .69 & $.05^{*}$ & 6.41 & .000 & 0.016 \\
\hline
\end{tabular}

$* p<.05$

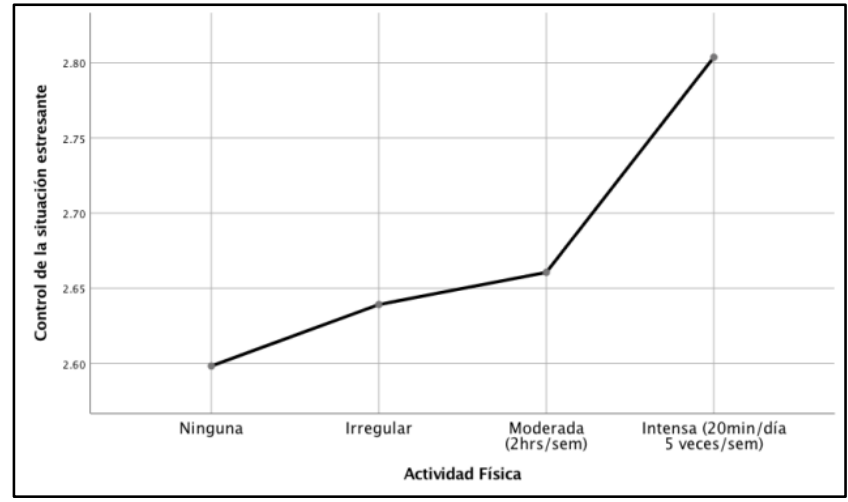

Figura 1. Promedio del control de la situación estresante en función de la actividad física

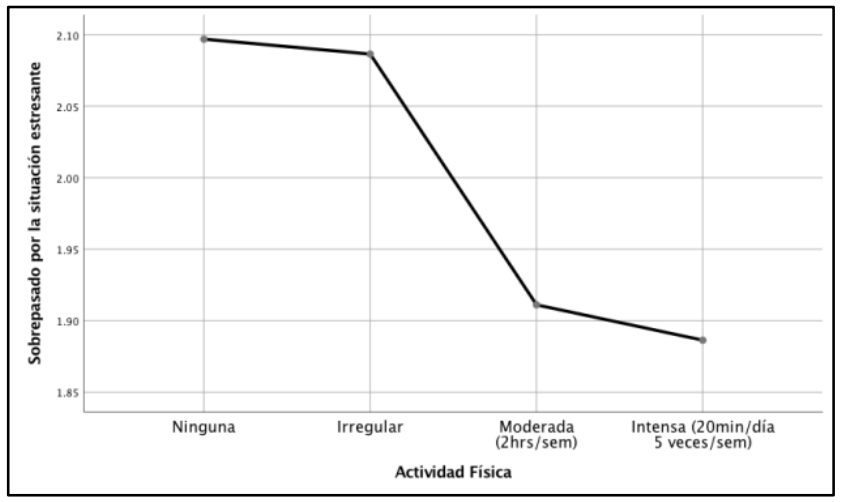

Figura 2. Promedio de sobrepasado por la situación estresante en función de la actividad física 


\section{Estrés, felicidad y actividad física en jóvenes universitarios}

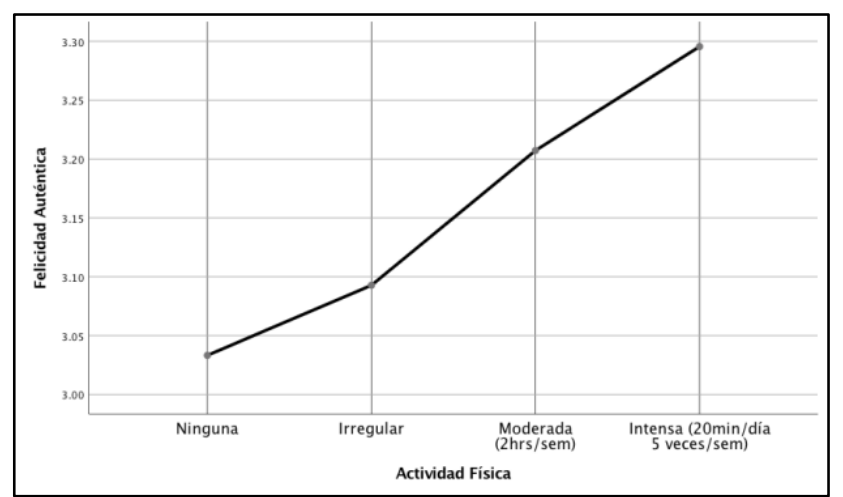

Figura 3. Promedio de la felicidad auténtica en función de la actividad física

\section{Análisis de mediación}

Se analizaron dos modelos de regresión mediada (M1 y M2), el primero (M1), considerando la percepción del estrés con control de la situación como predictora de la felicidad auténtica a través del nivel de actividad física (Tabla 3 y Figura 4); y el segundo (M2), considerando como predictor la percepción del estrés ante el sobrepaso de una situación (Tabla 4 y Figura $5)$.

Respecto al Modelo 1, la percepción de control ante una situación estresante resultó ser un predictor positivo del nivel de la AF, que, a su vez, predijo positivamente la felicidad auténtica (Figura 4). Se confirmó el papel mediador del nivel de AF en la relación entre el control de una situación estresante y la felicidad auténtica (Tabla 3), resultando una mediación parcial.

Tabla 3. Modelo 1. Nivel de AF como mediadora entre la percepción de control ante una situación estresante y la felicidad auténtica

\begin{tabular}{lccccc}
\hline VD / Predictor & $\mathrm{B}$ & $\mathrm{ET}$ & LI IC 95\% & LS IC 95\% & $\mathrm{R}_{2}$ \\
\hline Actividad Física & & & & & $.01^{* *}$ \\
$\quad \begin{array}{l}\text { Control de la situación } \\
\text { Felicidad Auténtica }\end{array}$ & .17 & .05 & .07 & .27 & $.31^{* *}$ \\
$\quad \begin{array}{l}\text { Actividad Física } \\
\quad \text { Control de la situación }\end{array}$ & .05 & .01 & .01 & .08 & .61 \\
\hline Efecto Indirecto [Mediador] & .56 & .02 & .50 & LS IC & \\
\hline [Actividad Física] & $\mathrm{B}$ & $\mathrm{ET}$ & $\begin{array}{c}\text { LI IC } \\
\text { Bootstrap 95\% }\end{array}$ & Bootstrap 95\% & .02 \\
\hline
\end{tabular}

Nota $:$ VD = variable dependiente; $\mathrm{B}=$ coeficiente de regresión no estandarizado; $\mathrm{ET}$ = error típico; $\mathrm{LI}=$ límite inferior; $\mathrm{LS}=$ límite superior; $\mathrm{IC}=$ intervalo de confianza. $* * p<.001$

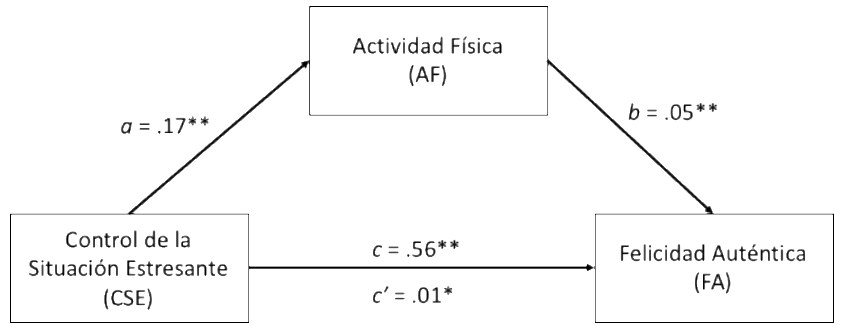

$$
\begin{gathered}
\mathrm{CSE} \rightarrow \mathrm{AF} \rightarrow \mathrm{FA} \\
\text { Efecto indirecto }(a b) \\
\mathrm{B}=.01, \mathrm{ET}=.00,95 \% \mathrm{IC}[.01, .02]
\end{gathered}
$$

Respecto al Modelo 2, la percepción del estrés sobrepasado por la situación estresante resultó ser un predictor negativo del nivel de la AF, y esta a su vez, igualmente predijo positivamente la felicidad auténtica (Figura 5). Se confirmó el papel mediador del nivel de AF en la relación entre la percepción del estrés sobrepasado por la situación estresante y la felicidad auténtica (Tabla 4), resultando una mediación parcial.

Figura 4. Representación gráfica del modelo de mediación del Control de la Situación Estresante $\rightarrow$

Actividad Física $\rightarrow$ Felicidad Auténtica 


\section{López-Walle, J., Tristán, J., Tomás, I., Gallegos-Guajardo, J., Gongora, E., y Hernández-Pozo, M. R.}

Tabla 4. Modelo 2. Nivel de AF como mediadora entre la percepción del estrés sobrepasado por la situación estresante y la felicidad auténtica

\begin{tabular}{lccccc}
\hline VD / Predictor & B & ET & LI IC 95\% & LS IC 95\% & $\mathrm{R}_{2}$ \\
\hline Actividad Física & & & & & $.01^{* *}$ \\
$\quad \begin{array}{l}\text { Sobrepasado por la situación } \\
\text { Felicidad Auténtica }\end{array}$ & -.17 & .04 & -.23 & -.07 & $.17^{* *}$ \\
$\quad \begin{array}{l}\text { Actividad Física } \\
\quad \text { Sobrepasado por la situación }\end{array}$ & .06 & .01 & .02 & .10 & \\
\hline $\begin{array}{l}\text { Efecto Indirecto [Mediador] } \\
\text { [Actividad Física] }\end{array}$ & -.36 & .02 & -.42 & LI IC & $\begin{array}{c}\text { LS IC } \\
\text { Bootstrap 95\% }\end{array}$ \\
\hline
\end{tabular}

Nota: VD = variable dependiente; $\mathrm{B}=$ coeficiente de regresión no estandarizado; $\mathrm{ET}$ = error típico; $\mathrm{LI}=$ límite inferior; $\mathrm{LS}=$ límite superior; $\mathrm{IC}=$ intervalo de confianza. ${ }^{* *} p<.001$

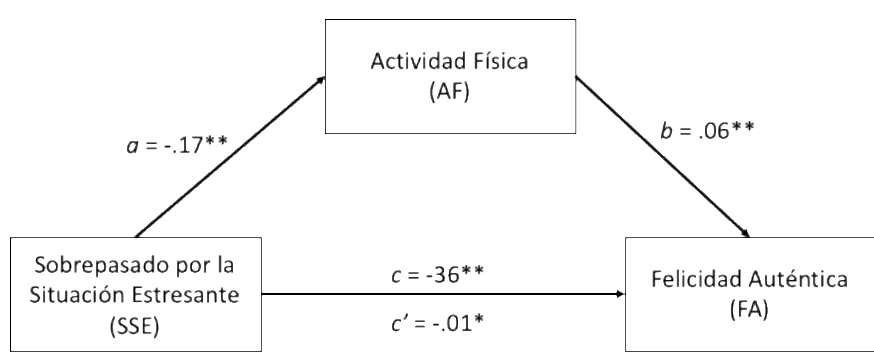

$$
\begin{gathered}
\mathrm{SSE} \rightarrow \mathrm{AF} \rightarrow \mathrm{FA} \\
\text { Efecto indirecto }(a b) \\
\mathrm{B}=-.01, \mathrm{ET}=.00,95 \% \mathrm{IC}[-.02,-.01]
\end{gathered}
$$

Figura 5. Representación gráfica del modelo de mediación de Sobrepasado por la Situación Estresante $\rightarrow$ Actividad Física $\rightarrow$ Felicidad Auténtica.

\section{DISCUSIÓN}

Considerando que la AF ha sido asociada con múltiples beneficios en la salud mental (Mang, 2013), y que se han encontrado diferencias entre los niveles de AF con respecto a la felicidad y al estrés (Opheim et al., 2011), el objetivo principal de este estudio fue: poner a prueba el papel de la práctica de $\mathrm{AF}$ en la reducción del estrés y el aumento de la felicidad, así como explorar el rol mediador de la actividad física (AF) en la interrelación de la percepción del estrés (con y sin control de la situación) y la felicidad auténtica. Los resultados obtenidos ofrecen apoyo a las diferentes hipótesis formuladas.

Respecto a las hipótesis 1 y 2 , se demuestra que el nivel de actividad física condiciona la percepción del estrés. Este resultado va en la línea de los resultados obtenidos por Opheim et al. (2011), sin embargo, en el presente estudio se aporta un enfoque diferente, ya que también se demuestra la perspectiva cognitiva de la percepción de estrés. Es decir, el percibirse con o sin control de la situación estresante difiere en función del nivel de actividad física, obteniendo medias mayores de percepción del control ante una situación estresante cuando el nivel de actividad física es intenso, y de forma inversa, encontrando medias mayores de percepción de sentirse sobrepasados por una situación estresante cuando no se realiza actividad física o se practica de forma irregular. Esto significa que es importante realizar actividad física moderada o intensa para percibir un mayor control de situaciones estresantes, o bien, no sentirse sobrepasados por dichas situaciones.

Respecto a las Hipótesis 1 y 3 , se confirma que la felicidad auténtica varía según el nivel de práctica de actividad física. Nuestros resultados coinciden con lo reportado en otros estudios (Opheim et al., 2011; Piqueras et al., 2011; Richards et al., 2015). De la misma manera se observó que mientras más actividad física se realice (más de 2 hrs por semana), y esta sea constante, se percibirá mayor felicidad.

La hipótesis 4 pretendía evaluar el papel mediador de la práctica de $\mathrm{AF}$ en la relación entre estrés percibido y felicidad auténtica. Al asociar las variables de percepción de estrés y felicidad auténtica, nuestros resultados aportan más allá de lo reportado en otros estudios (Schiffrin y Nelson, 2010; Terrill et al., 2014), quienes solamente consideran al estrés como una variable negativa, y por ende, este se relaciona de forma negativa con la felicidad auténtica. En esta investigación, se muestra que la percepción cognitiva del estrés marca la diferencia en dicha relación, siendo 


\section{Estrés, felicidad y actividad física en jóvenes universitarios}

una relación positiva entre la percepción del control de la situación estresante y la felicidad auténtica y, de forma contraria, una relación negativa entre sentirse sobrepasado por la situación estresante y la felicidad auténtica. Esto significa que, la forma en la cual se perciba la situación estresante (con o sin control) afectará de una u otra manera (positiva o negativamente) a la felicidad auténtica.

Además, se ha probado que el nivel de práctica de $\mathrm{AF}$ media la relación entre la percepción de la situación estresante (con o sin control) y la felicidad auténtica. Esto significa que, al percibir el control de una situación estresante se favorece la percepción de una felicidad auténtica, pero principalmente a través de un nivel de actividad física alta (moderada o intensa). De forma contraria, al percibirse sobrepasado ante una situación estresante, se impide la felicidad auténtica y esto principalmente con un nivel de actividad física baja, es decir, cuando no se realiza actividad física o se realiza de forma irregular.

Como limitante del estudio se considera que, al ser un estudio transversal, en el cual se aplicaron al mismo individuo tres instrumentos en un solo momento, no podemos suponer causalidad entre el estrés percibido (con y sin control de la situación estresante) y la felicidad auténtica, ni tampoco causalidad de cada una de estas variables con la $\mathrm{AF}$, únicamente el alcance logrado es mediante interrelaciones. Por lo cual, se sugiere para siguientes estudios poner a prueba modelos de tipo longitudinal, en donde tanto la percepción de estrés como la felicidad auténtica y la práctica de $\mathrm{AF}$ puedan actuar como predictoras y consecuentes, lo que reforzará aún más nuestras conclusiones sobre la posible causalidad.

\section{CONCLUSIONES}

Los resultados confirmaron las hipótesis del estudio, resaltando que aquellos estudiantes que indicaron mayores niveles de práctica de AF fueron también los que indicaron mayor percepción de control y menor sensación de sentirse sobrepasados ante una situación estresante, además de indicar mayor felicidad auténtica. Además, cuando las personas perciben control ante una situación estresante, realizan mayor práctica de AF, y pueden reportar mayores niveles de felicidad auténtica. Por el contrario, cuando se perciben sobrepasados por una situación estresante, presentan menores niveles de práctica de AF, y reportan menores niveles de felicidad auténtica. Es decir, dependerá más del control o no ante una situación estresante para impactar en la percepción de la felicidad. Además, se demuestra cómo la felicidad auténtica se relaciona con la percepción del estrés, siendo positiva cuando se percibe control ante una situación estresante, de lo contrario, al verse sobrepasado por la situación estresante, la relación sería negativa.

\section{APLICACIONES PRÁCTICAS}

Se recomienda diseñar programas de actividad física que propicien el mantenimiento de la actividad, con al menos 2 horas por semana; ya que eso permitirá impactar en el bienestar subjetivo de los individuos que la realicen y en sobreponerse de una mejor manera ante situaciones estresantes.

\section{REFERENCIAS (APA 6 ${ }^{\text {a EDICION) }}$}

1. Alarcón, R. (2006). Desarrollo de una Escala Factorial para Medir la Felicidad. Revista Interamericana de Psicologia, 40(1), 99-106.

2. Andreou, E., Alexopoulos, E. C., Lionis, C., Varvogli, L., Gnardellis, C., Chrousos, G. P., y Darviri, C. (2011). Perceived stress scale: reliability and validity study in Greece. International Journal of Environmental Research and Public Health, 8(8), 3287-3298. https://doi.org/10.3390/ijerph8083287

3. Campo-Arias, A., Bustos-Leiton, G. J., y RomeroChaparro, A. (2009). Consistencia interna y dimensionalidad de la Escala de Estrés Percibido (EEP-10 y EEP-14) en una muestra de universitarias de Bogotá, Colombia. Aquichan, 9(3), 271-280.

4. Cheng, H., y Furnham, A. (2001). Attributional style and personality as predictors of happiness and mental health. Journal of Happiness Studies, 2(3), 307-327.

5. Cohen, J. (1988). Statistical power analysis for the behavioral sciences. USA: Lawrence Erlbaum Associates. https://doi.org/10.2307/2136404

6. Cohen, S., Kamarck, T., y Mermelstein, R. (1983). A global measure of perceived stress. Journal of Health and Social Behavior, 24, 385-396. 


\section{López-Walle, J., Tristán, J., Tomás, I., Gallegos-Guajardo, J., Gongora, E., y Hernández-Pozo, M. R.}

7. Compton, W. C. (2005). Introduction to Positive Psychology. Belmont, CA, US: Thomson Wadsworth.

8. Dambrun, M., Desprès, G., y Lac, G. (2012). Measuring happiness: from fluctuating happiness to authentic-durable happiness. Frontiers in Psychology, $\quad 3, \quad 16$. https://doi.org/10.3389/fpsyg.2012.00016

9. Dias, C., Corte-Real, N., Corredeira, R., Barreiros, A., Bastos, T., y Fonseca, A. M. (2008). A prática desportiva dos estudantes universitários e suas relações com as autopercepções físicas, bem-estar subjectivo e felicidade. Estudos de Psicologia (Natal), 13(3), 223-232. https://doi.org/10.1590/S1413-

294X2008000300005

10. Diener, E. (2000). Subjective well-being: The science of happiness, and a proposal for a national index. American Psychologist, 55, 34-43. https://doi.org/10.1037//0003-066x.55.1.34

11. Diener, E., y Chan, M. Y. (2011). Happy people live longer: subjective well-being contributes to health and longevity. Applied Psychology Health and Well-Being, 3(1), 1-43. https://doi.org/10.1111/j.1758-

0854.2010.01045.x

12. Diener, E., Suh, E. M., Lucas, R. E., y Smith, H. L. (1999). Subjective well-being: Three decades of progress. Psychological Bulletin, 125(2), 276302. https://doi.org/10.1037//00332909.125.2.276

13. George, D., y Mallery, P. (2001). Reliability analysis. En SPSS for Windows step by step: A simple guide and reference (3rd ed., pp. 207-218). Needham Heights, MA: Allyn and Bacon. https://doi.org/10.4324/9781351033909

14. González, M. T., y Landero, R. (2007). Factor structure of the Perceived Stress Scale (PSS) in a sample from Mexico. The Spanish Journal of Psychology, 10, 199-206.

15. Hayes, A. F. (2013). Introduction to mediation, moderation, and conditional process analysis. New York, NY, USA: The Guilford Press.

16. Hernández-Pozo, M. R., López-Walle, J. M., Álvarez-Gasca, M. A., Romo-Gonzalez, T., Gallegos-Guajardo, J., Meza-Peña, C.,... Muñoz-
López, M. A. (en revisión-a). Niveles de estrés percibido en función de características sociodemográficas y de salud. Manuscrito enviado a dictamen para su publicación.

17. Hernández-Pozo, M. R., Góngora-Coronado, E. A., Romo-González, T., López-Walle, J. M., Meza-Peña, C., Álvarez-Gasca, M. A., ... Godínez-Vallejo, G. (en revisión-b). Tendencias de felicidad auténtica en mexicanos. Parte I: Indicadores de salud. Manuscrito enviado a dictamen para su publicación.

18. Hills, P., y Argyle, M. (2001). Emotional stability as a major dimension of happiness. Personality and Individual Differences, 31(8), 1357-1364. https://doi.org/10.1016/s0191-8869(00)00229-4

19. Jiménez-Moral, J. A., Zagalaz, M. L., Molero, D., Pulido-Martos, M., y Ruiz, J. R. (2013). Capacidad aeróbica, felicidad y satisfacción con la vida en adolescentes españoles. Revista de Psicología del Deporte, 22(2), 429-436.

20. King, L. A. (2001). The health benefits of writing about life goals. Personality and Social Psychology Bulletin, 27, 798-807. https://doi.org/10.1177/0146167201277003

21. Lee, E. H. (2012). Review of the psychometric evidence of the perceived stress scale. Asian Nursing Research, 6(4), 121-127. https://doi.org/10.1016/j.anr.2012.08.004

22. Mang, R. (2013). Physical Activity and Wellbeing: A Review of the Health Benefits of Physical Activity on Health Outcomes. Journal of Applied Medical Sciences, 2(2), 69-78.

23. Lyubomirsky, S., Sousa, L., y Dickerhoof, R. (2006). The costs and benefits of writing, talking, and thinking about life's triumphs and defeats. Journal of Personality and Social Psychology, 90, 692-708. https://doi.org/10.1037/00223514.90.4.692

24. Opheim, I. E., Eriksen, L., Moksnes, U. K., y Espnes, G. A. (2011). Stress and Happiness among Adolescents with Varying Frequency of Physical Activity. Perceptual and Motor Skills, 113(2), 631-646. https://doi.org/10.2466/02.06.10.13.pms.113.5.63 $1-646$ 


\section{Estrés, felicidad y actividad física en jóvenes universitarios}

25. Örücü, M. Ç., y Demir, A. (2009). Psychometric evaluation of perceived stress scale for Turkish university students. Stress and Health: Journal of the International Society for the Investigation of Stress, 25(1), 103-109. https://doi.org/10.1002/smi.1218

26. Piqueras, J. A., Kuhne, W., Vera-Villarroel, P., Van Straten, A., y Cuijpers, P. (2011). Happiness and health behaviours in Chilean college students: A cross-sectional survey. BMC Public Health, 11(1), 443. https://doi.org/10.1186/1471-2458$11-443$

27. Proyer, R.T., Gander, F., Wellenzohn, S., y Ruch, W. (2017). The Authentic Happiness Inventory Revisited: Addressing its Psychometric Properties, Validity, and Role in Intervention Studies. Journal of Well-Being Assessment, 1(1), 77-96. https://doi.org/10.1007/s41543-018-00060

28. Quick, J. C., y Quick, J. D. (2004). Healthy, happy, productive work: A leadership challenge. Organizational Dynamics, 33(4), 329-337. http://doi.org/10.1016/j.orgdyn.2004.09.001

29. Remor, E. (2006). Psychometric properties of a European Spanish version of the Perceived Stress Scale (PSS). The Spanish Journal of Psychology, 9(1), 86-93. https://doi.org/10.1017/S1138741600006004

30. Richards, J., Jiang, X., Kelly, P., Chau, J., Bauman, A., y Ding, D. (2015). Don't worry, be happy: cross-sectional associations between physical activity and happiness in 15 European countries. BMC Public Health, 15(1), 53. https://doi.org/10.1186/s12889-015-1391-4

31. Sanín-Posada, A., y Salanova, M. (2015). Análisis Psicométrico del Authentic Happiness Inventory (AHI) en población trabajadora Colombiana. Revista Interamericana de Psicología Ocupacional, 34(2), 75-83. https://doi.org/10.21772/ripo.v34n2a01

32. Seligman, M. (2011). La Autentica Felicidad. Barcelona: ZETA

33. Schiffrin, H. H., y Nelson, S. K. (2010). Stressed and happy? Investigating the relationship between happiness and perceived stress. Journal of Happiness Studies, 11(1), 33-39. https://doi.org/10.1007/s10902-008-9104-7
34. Scorsolini-Comin, F., Fontaine, A. M. G. V., Koller, S. H., y dos Santos, M. A. (2013). From authentic happiness to well-being: The flourishing of positive psychology. Psicologia: Reflexão $e$ Crítica, 26(4), 663-670. http://dx.doi.org/10.1590/S010279722013000400006

35. Seligman, M. (2013). Building the State of Wellbeing. Australia: Pearson.

36. Seligman, M. E. P., Steen, T. A., Park, N., y Peterson, C. (2005). Positive Psychology in progress. Empirical validation of interventions. American Psychologist, 60, 410-421. https://doi.org/10.1037/0003-066x.60.5.410

37. Shepherd, J., Oliver, M., y Schofield, G. (2015) Convergent validity and test-restest reliability of the authentic hapiness inventoy in working adults. Social Indicator Research, 124, 1049-1058. https://doi.org/10.1007/s11205-014-0812-6

38. Terrill, A. L., Müller, R., Jensen, M. P., Molton, I. R., Ipsen, C., y Ravesloot, C. (2014). Association between age, distress, and orientations to happiness in individuals with disabilities. Rehabilitation Psychology, 60(1), 27-35. https://doi.org/10.1037/rep0000016

39. Veenhoven, R., y Ehrhardt, J. (1995). The crossnational pattern of happiness: Test of predictions implied in three theories of happiness. Social Indicators Research, 34(1), 33-68. https://doi.org/10.1007/bf01078967

40. Zabihi, R., Ketabi, S., Tavakoli, M., y Ghadiri, M. (2014). Examining the internal consistency reliability and construct validity of the Authentic Happiness Inventory (AHI) among Iraninan EFL Learners. Current Psychology 33, 377-392. https://doi.org/10.1007/s12144-014-9217-6 